Pacific Journal of Mathematics

ON THE GENERALIZED PRINCIPAL IDEAL THEOREM AND David Fenimore Anderson, David Earl bobs,
Paul M. Eakins, Jr. and William James Heinz 


\title{
ON THE GENERALIZED PRINCIPAL IDEAL THEOREM AND KRULL DOMAINS
}

\author{
David F. Anderson, David E. Dobbs, \\ PAUl M. EAKIN, JR., AND William J. Heinzer
}

\begin{abstract}
If $R \subset T$ is an integral extension of domains and $R$ is Noetherian, then $T$ satisfies (the conclusion of the) generalized principal ideal theorem (or GPIT for short). An example is given of a twodimensional quasilocal domain $R$ satisfying GPIT such that the integral closure of $R$ is finite over $R$ but does not satisfy GPIT. If a commutative ring $R$ satisfies GPIT and an ideal $I$ of $R$ is generated by an $R$-sequence, then $R / I$ satisfies GPIT. If $R$ is a Noetherian domain and $G$ is a torsionfree abelian group, then $R[G]$ satisfies GPIT. An example is given of a three-dimensional quasilocal Krull domain that does not satisfy GPIT because its maximal ideal is the radical of a 2-generated ideal.
\end{abstract}

1. Introduction. All rings considered in this paper are commutative with identity. Let $R$ be a ring. As in [4], we say that $R$ satisfies PIT (for "principal ideal theorem") if $h t(P) \leq 1$ for each prime ideal $P$ of $R$ which is minimal over a principal ideal of $R$. According to Krull's Hauptidealsatz, which has been called "the most important single theorem in the theory of Noetherian rings" by Kaplansky [12, p. 104], each Noetherian ring satisfies PIT. So does each Krull domain. A generalization of this fact has been noted by Davis [5, p. 182]. Additional examples of rings satisfying PIT were obtained in [4] (see especially [4, Corollary 3.5, Theorem 3.10, Corollary 3.11 and Theorem 6.5]). Much of [4] addressed the stability of "satisfies PIT" under various ring-theoretic passages. The purpose of this paper is to study similar questions concerning GPIT (for "generalized principal ideal theorem"). By definition, a ring $R$ satisfies GPIT if $h t(P) \leq n$ for each prime ideal $P$ of $R$ which is minimal over an $n$-generated ideal of $R$.

According to Krull's generalized principal ideal theorem [12, Theorem 152] (also known as Krull's altitude theorem [13, Theorem 9.3]), each Noetherian ring satisfies GPIT. However, not every Krull domain satisfies GPIT. The first example of this phenomenon seems to be the one discovered by Eakin and Heinzer in 1969 after further analysis of their work in [6] concerning a construction of Rees [14]. Later 
examples of this phenomenon have appeared in the literature (cf. [7]). For instance, Anderson and Mulay were stimulated to develop [1] in the summer of 1987 after being shown the details of the Eakin-Heinzer example. That example, now nearly twenty years old, is developed in Example 5.1. This work gives as a bonus (see Example 5.2) an example of an (integral) domain $R$ satisfying PIT such that $R / P$ does not satisfy PIT for some principal prime ideal $P$ of $R$. Example 5.2 relates to the theme of $\S 3$, namely the behavior of GPIT under homomorphic images. The most noteworthy result in this section, Theorem 3.3 , establishes that $R / I$ satisfies GPIT if $R$ satisfies GPIT and $I$ is an ideal of $R$ which is generated by an $R$-sequence.

Section 2 concerns the behavior of GPIT under integral extensions. A positive result, Corollary 2.3, is available in the Noetherian case: if $R \subset T$ is an integral extension of domains and $R$ is Noetherian, then $T$ satisfies GPIT. This result is of interest for several reasons discussed in $\S 2$. For instance, an example of Nagata [13] shows that such $T$ need not be Noetherian even if $T$ is an overring of $R$ and $\operatorname{dim}(R)=2$. On the other hand, Example 2.4 shows, in the absence of the Noetherian hypothesis, that the conclusion of Corollary 2.3 fails, even if $T$ is finite over $R$ and $\operatorname{dim}(R)=2$. One moral is that GPIT does not satisfy the Noetherian-like stability typified by the Hilbert Basis Theorem.

Section 4 concerns monoid domains. Its principal application, Corollary 4.3, includes the following assertion. If $R$ is a Noetherian domain and $G$ a torsionfree abelian group, then $R[G]$ satisfies GPIT. As Example 4.5 shows, the results are qualitatively different if $G$ is replaced by an arbitrary abelian monoid.

It will be convenient to follow [12, p. 40], denoting the lying-over, going-up, going-down and incomparability properties by LO, GU, GD and INC, respectively. The radical of an ideal $I$ of a ring $R$ will be denoted by $\operatorname{rad}_{R}(I)$, or $\operatorname{rad}(I)$ if no confusion can result. The integral closure of a ring $R$ will be denoted by $R^{\prime}$. Other notation will be standard, as in [12], [13].

2. GPIT and integrality. It is easy to verify that "satisfies GPIT" is a local property. In other words, a domain $R$ satisfies GPIT if and only if $R_{M}$ satisfies GPIT for each maximal ideal $M$ of $R$. Studying such results on the passage of "satisfies GPIT" is our main concern, with this section focused on integral extensions. We begin with a result stated in [4, Remark 5.3(a)]. Its proof is an easy adaptation of the proofs of [4, Proposition 5.1 and Corollary 5.2] and is hence omitted. 
Proposition 2.1. Let $R \subset T$ be an extension of domains. Then:

(a) Suppose $R \subset T$ satisfies LO, INC and GD. If $T$ satisfies GPIT, then $R$ satisfies GPIT.

(b) Suppose $R \subset T$ satisfies $G U$ and INC. If $T$ satisfies GPIT, then $R$ satisfies GPIT.

(c) If $T$ is integral over $R$ and $T$ satisfies GPIT, then $R$ satisfies GPIT.

Notice that a two-dimensional domain satisfies GPIT if (and only if) it satisfies PIT. Of course, each domain of dimension $\leq 1$ satisfies GPIT. Accordingly, two-dimensional domains receive special attention below.

It seems natural to ask about the converse of Proposition 2.1(c). In other words, if $R \subset T$ is an integral extension of domains and $R$ satisfies GPIT, must $T$ also satisfy this property? Corollary 2.3 gives an affirmative answer in case $R$ is Noetherian. Thus, (any Krull domain arising as) the integral closure of a Noetherian domain satisfies GPIT. Also, by taking integral closure in a suitable infinite-dimensional field extension, we can find an integrally closed non-Krull domain satisfying GPIT. Moreover, although a famous example of Nagata [13, Example 4, p. 207] shows that an integral overring $T$ of a two-dimensional Noetherian domain $R$ need not be Noetherian, Corollary 2.3 assures that any such $T$ must satisfy GPIT. However, as Example 2.4 reveals, in the absence of the Noetherian hypothesis, "satisfies (G)PIT" fails to ascend from $R$ to $T$, even if $\operatorname{dim}(R)=2$.

THEOREM 2.2. For a domain $R$, the following conditions are equivalent:

(1) If $u_{1}, \ldots, u_{n}$ are finitely many elements of a domain which contains $R$ and is integral over $R$, then $R\left[u_{1}, \ldots, u_{n}\right]^{\prime}$ satisfies GPIT;

(2) If $R$ is a subring of a domain $T$ which is integral over $R$, then $T$ satisfies GPIT.

Proof. (2) $\Rightarrow(1)$ trivially. Suppose that the converse fails. Then there exists $P \in \operatorname{Spec}(T)$ such that $P$ is minimal over some $n$ generated ideal $J$ of $T$ and $h t(P)>n$. Choose an $n$-generating set $\left\{u_{i}\right\}$ for $J$. We may replace $R$ with $R\left[u_{1}, \ldots, u_{n}\right]$. We thus have $J=I T$, for the $n$-generated ideal $I=\sum R u_{i}$ of $R$. 
Pick a chain $P=P_{0} \supset P_{1} \supset \cdots \supset P_{n+1}$ of distinct primes in $T$. Consider the ring $S=R^{\prime} T$ (inside the quotient field of $T$ ). Evidently, $S$ is integral over $T$. Applying LO and GU, we have a chain $N=N_{0} \supset \cdots \supset N_{n+1}$ of primes in $S$ lying over the given chain $\left\{P_{i}\right\}$ in $T$. Moreover, since $T \subset S$ satisfies INC, we have $N$ minimal over $\sum S u_{i}=I S=J S$. Put $Q_{i}=N_{i} \cap R^{\prime}$ in $\operatorname{Spec}\left(R^{\prime}\right)$. Since $S$ is integral over $R^{\prime}$, it follows via INC that $\left\{Q_{i}\right\}$ consists of $n+2$ distinct primes of $R^{\prime}$, whence $h t\left(Q_{0}\right)>n$. Next, observe via the classical result of Krull (cf. [13, Theorem 10.13]) that $R^{\prime} \subset S$ satisfies GD. As $N$ is minimal over $\sum S u_{i}$, it now follows via GD that $Q_{0}$ is minimal over $\sum R^{\prime} u_{i}$. In particular, $R^{\prime}$ does not satisfy GPIT, the desired contradiction to (1).

The proof of Theorem 2.2 also establishes the following assertion. Let $R \subset T$ be an integral extension of domains. If $R\left[u_{1}, \ldots, u_{n}\right]^{\prime}$ satisfies GPIT for each finite subset $\left\{u_{i}\right\}$ of $T$, then $T$ satisfies GPIT.

COROllary 2.3. If $R \subset T$ is an integral extension of domains and $R$ is Noetherian, then $T$ satisfies GPIT.

Proof. By Theorem 2.2, it suffices to show that $R\left[u_{1}, \ldots, u_{n}\right]^{\prime}$ satisfies GPIT if $u_{1}, \ldots, u_{n}$ are integral elements of a domain containing $R$. As $R\left[u_{1}, \ldots, u_{n}\right]$ is Noetherian (by the Hilbert Basis Theorem), we may replace $R$ with $R\left[u_{1}, \ldots, u_{n}\right]$. Thus, our task is reduced to showing that $T=R^{\prime}$ satisfies GPIT.

Without loss of generality, $R$ is local. Hence, by [11, Chapitre 0 , Corollaire 23.2 .5$, p. 218], $R$ has a finitely generated (integral) overring $S$ such that the canonical map $\operatorname{Spec}(T) \rightarrow \operatorname{Spec}(S)$ is injective (in fact, radiciel). Since $S$ is Noetherian and $T=S^{\prime}$, we may replace $R$ with $S$.

Consider a prime ideal $P$ of $T$ such that $P$ is minimal over a finitely generated ideal $\left(v_{1}, \ldots, v_{k}\right)$ of $T$. Put $A=R\left[v_{1}, \ldots, v_{k}\right]$. Since $R \subset A \subset T$ and $\operatorname{Spec}(T) \rightarrow \operatorname{Spec}(R)$ is injective, it follows that $\operatorname{Spec}(T) \rightarrow \operatorname{Spec}(A)$ is injective. As $A \subset T$ also satisfies LO and GU by integrality, it now follows easily that $A \subset T$ satisfies GD. Hence, $Q=P \cap A$ is minimal over $\sum A v_{i}$. Since $A$ is Noetherian, $A$ satisfies GPIT by Krull's altitude theorem ([12, Theorem 152], [13, Theorem 9.3]), and so $h t(Q) \leq k$. But $h t(P) \leq h t(Q)$ since $A \subset T$, being integral, satisfies INC. Thus $h t(P) \leq k$, whence $T$ satisfies GPIT. 
EXAMPLE 2.4. There exists a quasilocal two-dimensional domain $R$ satisfying GPIT and an element $u$ such that $R^{\prime}=R[u]$ and $R^{\prime}$ does not satisfy $(\mathrm{G}) \mathrm{PIT}$.

Proof. Let $X, Y$ be algebraically independent indeterminates over a field $k$. Put $D=k[X, Y]_{(X-1, Y-1)}$. Then $D=k+M_{1}$, where $M_{1}=(X-1, Y-1) D$ is the unique maximal ideal of $D$. Next, consider the rank 2 valuation $v$ of $k(X, Y)$ over $k$, with value group $\mathbb{Z} \oplus \mathbb{Z}$ lexicographically ordered, determined as follows. Put $v(X)=(1,0)$ and $v(Y)=(0,1)$. It follows that the value of $v$ at any nonzero polynomial in $k[X, Y]$ is the infimum of the values of its constituent monomials. The associated valuation ring is $V=$ $k[Y]_{(Y)}+X k[X, Y]_{(X)}$, which can be written as $V=k+M_{2}$, where $M_{2}=Y V$ is the maximal ideal of $V$. Put $J=M_{1} \cap M_{2}$ and $R=$ $k+J$. We shall show that $R$ has the asserted properties.

We shall first show $R^{\prime}=D \cap V$. (The following proof is inspired by that of [10, Example 4.3].) Since $D$ and $V$ are each integrally closed and contain $R$, it is clear that $R^{\prime} \subset D \cap V$. For the reverse inclusion, it suffices to show that each $t \in D \cap V$ is integral over $R$. (To be sure, one should also observe that $D \cap V$ is an overring of $R$; for this, just notice that $J$ is a common nonzero ideal of $D \cap V$ and $R$.) Now, write $t=a_{1}+m_{1}=a_{2}+m_{2}$, with $a_{i} \in k$ and $m_{i} \in M_{i}$. Then $\left(t-a_{1}\right)\left(t-a_{2}\right)=m_{1} m_{2} \in M_{1} \cap M_{2}=J \subset R$, and so $t$ is a root of the monic polynomial $Z^{2}+\left(-a_{1}-a_{2}\right) Z+a_{1} a_{2}-m_{1} m_{2} \in R[Z]$. It follows that $R^{\prime}=D \cap V$.

Next, we claim that $R$ is quasilocal, with unique maximal ideal $J$. To see this, argue as in [13, E2.1, p. 204]: if $r \in J$, then $(1+r)^{-1}-1=$ $-r(1+r)^{-1} \in J$ and so $(1+r)^{-1} \in 1+J$. Hence $J$, which is obviously maximal in $R$, is contained in the Jacobian radical of $R$, proving the claim.

Consider the primes $P_{1}=M_{1} \cap R^{\prime}$ and $P_{2}=M_{2} \cap R^{\prime}$. As each of these meets $R$ in $J$, it follows via integrality that $P_{1}$ and $P_{2}$ are maximal in $R^{\prime}$. Moreover, they are distinct, since $X \in P_{2} \backslash P_{1}$. We claim next that $R_{P_{1}}^{\prime}=D$ and $R_{P_{2}}^{\prime}=V$. Now, for any multiplicatively closed subset $S$ of $R^{\prime}$, we have $R_{S}^{\prime}=D_{S} \cap V_{S}$. Hence, with $S_{i}$ denoting $R^{\prime} \backslash P_{i}$, the claims will follow if we show that $V_{S_{1}}=k(X, Y)$ and $D_{S_{2}}=k(X, Y)$.

Consider any nonzero $g \in k[X, Y]$. If $v(g)=(i, j)$, then $v\left(g\left(X^{i} Y^{j}\right)^{-1}\right)=(i, j)-(i, 0)-(0, j)=0$, whence $g V=X^{i} Y^{j} V$. It follows easily that $V\left[(X Y)^{-1}\right]=k(X, Y)$. As $X Y \in R^{\prime} \backslash P_{1}=S_{1}$, 
we have $V_{S_{1}}=k(X, Y)$. Moreover, for $g$ as above,

$$
h=g\left(X^{i} Y^{j}\right)^{-1} \in\left(V \backslash M_{2}\right) \cap D=R^{\prime} \backslash P_{2}=S_{2} .
$$

Since $\left(X^{i} Y^{j}\right)^{-1} \in D$, we have $g^{-1}=\left(X^{i} Y^{j}\right)^{-1} h^{-1} \in D_{S_{2}}$. It follows that $D_{S_{2}}=k(X, Y)$, and the claims have been established.

Since $V$ is a two-dimensional valuation domain, $V$ does not satisfy PIT. As $R_{P_{2}}^{\prime}=V$, it follows from [4, Proposition 3.1(a)] that $R^{\prime}$ does not satisfy PIT.

Notice that $R \neq R^{\prime}$ since $X \in R^{\prime} \backslash R$. Moreover, $X$ satisfies a quadratic integrality equation over $R$, as $X(X-1) \in M_{1} \cap M_{2}=J \subset$ $R$. We claim that $R[X]=R^{\prime}$. (In other words, $u=X$ satisfies the assertion.) To see this, first note that the equality $R_{P_{1}}^{\prime}=D$ leads to canonical isomorphisms $k \stackrel{\cong}{\rightrightarrows} D / M_{1} \cong R^{\prime} / P_{1}$, whence $R^{\prime}=k+P_{1}$. Similarly, $R_{P_{2}}^{\prime}=V$ leads to $k \stackrel{\cong}{\rightrightarrows} V / M_{2} \cong R^{\prime} / P_{2}$, whence $R^{\prime}=k+P_{2}$. Since $X P_{1} \subset X M_{1} \subset M_{1}$ and $X P_{1} \subset M_{2} V=M_{2}$, we have $X P_{1} \subset J$, so that

$$
X R^{\prime}=X\left(k+P_{1}\right)=X k+X P_{1} \subset X R+J \subset R[X] .
$$

As $X-1 \in R^{\prime} \cap M_{1}=P_{1}$ and

$$
P_{1} P_{2} \subset P_{1} \cap P_{2}=M_{1} \cap M_{2} \cap R^{\prime}=J \cap R^{\prime}=J,
$$

we also have

$$
\begin{aligned}
(X-1) R^{\prime} & =(X-1)\left(k+P_{2}\right) \\
& =(X-1) k+(X-1) P_{2} \subset R[X]+P_{1} P_{2}=R[X] .
\end{aligned}
$$

Hence, $R^{\prime} \subset X R^{\prime}+(X-1) R^{\prime} \subset R[X]$; that is, $R[X]=R^{\prime}$, as claimed.

Since $R^{\prime}$ is integral over $R$, any maximal ideal $M$ of $R^{\prime}$ meets $R$ in $J=M_{1} \cap M_{2}=P_{1} \cap P_{2}$. It follows that $M$ contains (and hence equals) one of $P_{1}, P_{2}$. Thus, $P_{1}$ and $P_{2}$ are all the maximal ideals of $R^{\prime}$, and so

$$
\operatorname{dim}\left(R^{\prime}\right)=\sup \left\{\operatorname{dim}\left(R_{P_{1}}^{\prime}\right), \operatorname{dim}\left(R_{P_{2}}^{\prime}\right)\right\}=\sup \{\operatorname{dim}(D), \operatorname{dim}(V)\}=2 .
$$

By integrality, $\operatorname{dim}(R)=\operatorname{dim}\left(R^{\prime}\right)=2$.

It remains only to show that $R$ satisfies GPIT. As $\operatorname{dim}(R)=2$, we need only show that $R$ satisfies PIT, namely that $J$ is not minimal over any nonzero principal ideal $R s$. Consider $s \in J \backslash\{0\}$. In the (Noetherian) ring $D$, the prime $P_{1} D$ has height 2 and contains $s$, but cannot be minimal over $D s$ (by Krull's altitude theorem). Hence $D s \subset Q D \varsubsetneqq P_{1} D$ for some prime $Q$ of $R^{\prime}$ properly contained in $P_{1}$. 
By INC, we have $Q \cap R \varsubsetneqq P_{1} \cap R=J$. As $s \in Q D \cap R=Q \cap R, J$ is not minimal over $R s$.

To close the section, we record some additional results on the passage of "satisfies GPIT" that are analogous to results in [4].

Proposition 2.5. (a) Let $R$ and $T$ be subrings of a field $K$ such that $\operatorname{Spec}(R)=\operatorname{Spec}(T)$ as sets. Then $R$ satisfies GPIT if and only if $T$ satisfies GPIT.

(b) Let $T$ be a quasilocal domain with nonzero maximal ideal $M$ and residue field $k=T / M$, let $\pi: T \rightarrow k$ denote the canonical surjection, and let $D$ be a subring of $k$. Then $\pi^{-1}(D)$ satisfies GPIT if and only if $D$ is a field and $T$ satisfies GPIT.

Proof. The proof of (a) follows that of [4, Corollary 3.2(b)] provided that $R \subset T$. One reduces to this case (assuming, without loss of generality, that $R$ is not a field) by considering the unique smallest subring of $R \cap T$ that has the same spectrum as $R$ and $T$. (Cf. [3, Proposition 2.3].) As for (b), argue as in the proof of [4, Corollary $3.2(\mathrm{c})$ ], with (a) now playing the role of [4, Corollary $3.2(\mathrm{~b})$ ].

3. GPIT and homomorphic images. We begin this section with a simple sufficient condition for a ring to satisfy GPIT (cf. [12, Exercise 6, p. 114]).

Proposition 3.1. If $R$ is a ring such that $R / P$ satisfies GPIT for each minimal prime ideal $P$ of $R$, then $R$ satisfies GPIT.

Proof. Suppose the assertion fails. Then there exists $Q \in \operatorname{Spec}(R)$ such that $Q$ is minimal over some $n$-generated ideal $I$ of $R$ and $h t(Q)>n$. Pick a chain

$$
Q=P_{0} \supset P_{1} \supset \cdots \supset P_{n+1}=P
$$

of distinct primes in $R$. Without loss of generality, $P$ is a minimal prime. Since the ring $R / P$ satisfies GPIT and its prime $\bar{Q}=Q / P$ is minimal over the $n$-generated ideal $(I+P) / P$, it follows that $h t(\bar{Q}) \leq$ $n$. However, the chain $\left\{P_{i} / P\right\}$ of distinct primes reveals that $h t(\bar{Q})>$ $n$, the desired contradiction.

It is natural to ask whether a variation of the converse of Proposition 3.1 is valid. In this regard, it was claimed in [4, Remark 5.3(a)] that if $R$ satisfies GPIT, then so does $R / I$ for each finitely generated ideal $I$ of $R$. The authors of [4] now view this claim as suspect, and 
hereby retract it. A weaker positive result will be obtained in Theorem 3.3. Before this, we give an easy variant of Proposition 3.1.

Proposition 3.2. A ring $R$ satisfies GPIT if and only if $R_{\text {red }}$ satisfies GPIT.

Proof. The proof follows from the next three observations. The chains of distinct primes in $R$ are in one-to-one correspondence with the chains of distinct primes in $R_{\text {red, }}$, with the chain $P_{0} \supset \cdots \supset$ $P_{n+1}$ in $R$ corresponding to the chain $\left\{P_{i} / \operatorname{rad}(R)\right\}_{i=0}^{n+1}$ in $R_{\text {red }}$; thus, $h t\left(P_{0}\right)=h t\left(P_{0} / \operatorname{rad}(R)\right)$; and $P_{0} / \operatorname{rad}(R)$ is minimal over an $n$ generated ideal $J$ of $R_{\text {red }}$ if and only if $J$ can be written as $J=$ $(I+\operatorname{rad}(R)) / \operatorname{rad}(R)$, where $I$ is an $n$-generated ideal of $R$ such that $P_{0}$ is minimal over $I$.

THEOREM 3.3. If a ring $R$ satisfies GPIT and an ideal $I$ of $R$ is generated by an $R$-sequence, then $R / I$ satisfies GPIT.

It will be convenient to make the following definition and remarks before proving Theorem 3.3. If $R$ is a ring and $n$ is a nonnegative integer, we shall say that $R$ satisfies $n$-PIT in case $h t(P) \leq n$ for each $P \in \operatorname{Spec}(R)$ which is minimal over an $n$-generated ideal of $R$. Evidently, each ring $R$ satisfies 0-PIT and $n$-PIT for all $n \geq$ $\operatorname{dim}(R)$; 1-PIT is equivalent to PIT; and $R$ satisfies GPIT if and only if $R$ satisfies $n$-PIT for each $n \geq 0$. It is easy to see from the above proofs that Propositions 2.1, 2.5(a), 3.1 and 3.2 all remain valid if one replaced "GPIT" with " $n$-PIT" in their statements. Such generalizations aside, our purpose in introducing $n$-PIT is to develop the next result, which immediately implies Theorem 3.3.

LEMMA 3.4. Let $R$ be a ring satisfying $k$-PIT for some $k>0$. Let $I$ be an ideal of $R$ generated by an $R$-sequence $y_{1}, \ldots, y_{n}$, for some $n<k$. Then $\bar{R}=R / I$ satisfies $(k-n)-P I T$.

Proof. We shall proceed by induction on $n$, which clearly can be assumed positive.

Case $n=1$ : Since $y=y_{1}$ is a non-zerodivisor, $y$ lies in no minimal prime of $R$ (cf. [12, Theorem 84]). Suppose the assertion fails. Then there exists a prime $P / I$ of $\bar{R}$ such that $h t(P / I)>k-1$ and $P / I$ is minimal over $\left(\overline{x_{1}}, \ldots, \overline{x_{k-1}}\right)$. (As usual, if $x \in R$, then $\bar{x}$ denotes $x+(y) \in \bar{R}$.) Hence there is a chain $P \supset P_{1} \supset \cdots \supset P_{k}$ 
of distinct primes in $R$ containing $y$. As noted above, $y$ is in no minimal prime. Thus, being nonminimal, $P_{k}$ properly contains a minimal prime of $R$. It follows that $h t(P) \geq k+1$. However, $h t(P) \leq$ $k$ since $R$ satisfies $k$-PIT and $P$ is minimal over the $k$-generated ideal $\left(x_{1}, \ldots, x_{k-1}, y\right)$. This (desired) contradiction establishes the induction basis.

Induction step: By the induction hypothesis, $A=R /\left(y_{1}, \ldots, y_{n-1}\right)$ satisfies $(k-(n-1))$-PIT; that is, $(k-n+1)$-PIT. Consider $z=$ $y_{n}+\left(y_{1}, \ldots, y_{n-1}\right) \in A$. Since $y_{1}, \ldots, y_{n}$ is an $R$-sequence, $z$ is a non-zerodivisor in $A$. It follows from the proof of the "case $n=1$ " that $A /(z)$ satisfies $((k-n+1)-1)$-PIT; that is, $(k-n)$-PIT. Since $A /(z) \cong \bar{R}$, the proof of Lemma 3.4 (and hence of Theorem 3.3) is complete.

4. GPIT and monoid domains. In this section, we study when a monoid domain $R[S]$ satisfies GPIT. ([8] is an excellent reference for monoid domains.) We show in Corollary 4.3 that any torsionfree abelian group ring over a Noetherian domain satisfies GPIT, and next give an example to show that "almost anything" can happen for a monoid domain. For simplicity, we assume that all coefficient rings in this section are domains. We begin with a result noted in the remarks preceding [4, Proposition 6.4]; its proof is omitted.

Proposition 4.1. Let $R$ be a domain. Then $R\left[\left\{X_{\alpha}\right\}\right]$ satisfies GPIT for each family $\left\{X_{\alpha}\right\}$ of algebraically independent indeterminates if and only if the polynomial ring $R\left[X_{1}, \ldots, X_{n}\right]$ satisfies GPIT for each positive integer $n$.

In general, $R[X]$ need not satisfy GPIT when $R$ satisfies GPIT (cf. [4, Remark 6.2]). However, if $R[X]$ satisfies GPIT, than by Theorem 3.3, $R \cong R[X] /(X)$ also satisfies GPIT. Our next result, the main one of this section, shows that "GPIT-stability" (in the sense of Proposition 4.1) characterizes the validity of GPIT for certain group rings over $R$.

THEOREM 4.2. Let $G$ be a nonzero torsionfree abelian group with $n=\operatorname{rank}(G)$ finite. Let $R$ be a domain. Then the following conditions are equivalent:

(1) $R\left[X_{1}, \ldots, X_{n}\right]$ satisfies $G P I T$;

(2) $R\left[X_{1}, X_{1}^{-1}, \ldots, X_{n}, X_{n}^{-1}\right]$ satisfies GPIT;

(3) $R[G]$ satisfies $G P I T$. 
Proof. (1) $\Rightarrow(2)$ since GPIT is preserved by localization.

(2) $\Rightarrow(3)$ : Suppose that $B=R[G]$ does not satisfy GPIT. Then there exist $f_{1}, \ldots, f_{r} \in B$ and a prime ideal $P$ of $B$ with $h t(P)>r$ such that $P$ is minimal over $\left(f_{1}, \ldots, f_{r}\right) B$. Choose a finitely generated subgroup $F$ of $G$ with $\operatorname{rank}(F)=n$ such that $f_{1}, \ldots, f_{r} \in A=$ $R[F]$ and $Q=P \cap A$ has $h t(Q)>r$. Since $A$ satisfies GPIT by (2), there exists a prime ideal $Q_{1}$ of $A$ with $\left(f_{1}, \ldots, f_{r}\right) A \subset Q_{1} \varsubsetneqq Q$. As $B$ is a flat (in fact, free [8, Theorem 12.1]) $A$-module, $A \subset B$ satisfies $G D$ (cf. [12, Exercise 37, p. 44]). Thus there is a prime ideal $P_{1}$ of $B$ with $\left(f_{1}, \ldots, f_{r}\right) B \subset P_{1} \varsubsetneqq P$, contradicting the minimality of $P$.

$(3) \Rightarrow(2)$ : Choose a finitely generated subgroup $F$ of $G$ with $\operatorname{rank}(F)=n$. Hence $G / F$ is a torsion group, and thus

$$
A=R\left[X_{1}, X_{1}^{-1}, \ldots, X_{n}, X_{n}^{-1}\right] \cong R[F] \subset R[G]
$$

is an integral extension. By Proposition 2.1(c), $A$ satisfies GPIT.

$(2) \Rightarrow(1)$ : Suppose that a prime ideal $P$ of $A=R\left[X_{1}, \ldots, X_{n}\right]$ is minimal over $I=\left(f_{1}, \ldots, f_{r}\right)$. We show that $h t(P) \leq r$. By applying a suitable automorphism, $f\left(X_{1}, \ldots, X_{n}\right) \mapsto f\left(X_{1}+a_{1}, \ldots, X_{n}+a_{n}\right)$ of $A$, we may assume that $X_{i} \notin P$ for $1 \leq i \leq n$. Let $B=$ $R\left[X_{1}, X_{1}^{-1}, \ldots, X_{n}, X_{n}^{-1}\right]$. Then $P B$ is minimal over $I B$ since $B$ is a ring of fractions of $A$. Thus $h t(P B) \leq r$ since $B$ satisfies GPIT. But $h t(P)=h t(P B)$.

COROllary 4.3. Let $R$ be a domain. Then $R[G]$ satisfies GPIT for each torsionfree abelian group $G$ if and only if $R\left[X_{1}, \ldots, X_{n}\right]$ satisfies GPIT for each positive integer $n$. In particular, if $R$ is $a$ domain containing, and integral over, a Noetherian domain, then $R[G]$ satisfies GPIT for each torsionfree abelian group or free abelian monoid $G$.

Proof. $(\Rightarrow)$ follows directly from Theorem 4.2. Conversely, suppose that $R[G]$ does not satisfy GPIT, for some torsionfree abelian group $G$. Arguing as in the proof of $(2) \Rightarrow(3)$ of Theorem 4.2, we see that $R[F]$ does not satisfy GPIT for some finitely generated subgroup $F$ of $G$ with $\operatorname{rank}(F)=n$. But this contradicts $(1) \Rightarrow(2)$ of Theorem 4.2 because $R\left[X_{1}, \ldots, X_{n}\right]$ satisfies GPIT by hypothesis.

The "in particular" statement follows from the first assertion and Corollary 2.3 for the group ring case, and from Proposition 4.1 and Corollary 2.3 for the free abelian monoid case. 
REMARKS 4.4. (a) It is worthwhile to note, via Theorem 3.3 and Theorem 4.2, that if $R\left[X_{1}, \ldots, X_{n}\right]$ satisfies GPIT, then $R[G]$ satisfies GPIT for each torsionfree abelian group $G$ with $\operatorname{rank}(G) \leq n$.

(b) Corollary 4.3 may be used to construct additional examples of non-Noetherian domains which satisfy GPIT. In particular, the group ring $k[G]$ satisfies GPIT for any field $k$ and torsionfree abelian group $G$. This construction has been used to construct finite-dimensional non-Noetherian UFD's [9, Theorem 2] and [8, §14]). (Recall that $k[G]$ is a Krull domain $\Leftrightarrow k[G]$ is a UFD $\Leftrightarrow$ each ring-one subgroup of $G$ is cyclic [8, Theorems $16.2,14.7,14.10$, and 14.15].) Thus, the non-Noetherian Krull domains constructed as group rings over a field satisfy GPIT.

(c) Finally, we note that Proposition 4.1 and Theorem 4.2 have $n$-PIT analogues; we leave these to the interested reader.

It is natural to ask if monoid domains other than polynomial rings and group rings satisfy GPIT. Our next example shows that the answer may be negative even for a very nice domain (cf. [12, Exercise 8, p. 114] and [4, Remark 3.6(c)]).

EXAMPLE 4.5. Let $k$ be a field and $1 \leq m<n \leq \infty$. Set $T=$ $k\left[\left\{X_{i}\right\}_{i=1}^{n}\right], P=\left(X_{1}, \ldots, X_{m}\right) T$, and $R=k+P$. Then $R=k[S]$, where $S$ is the submonoid of $\mathbb{Z}_{+}^{n}$ determined by the exponents of the monomials in $R$. Moreover:

(1) $\operatorname{dim}(R)=\operatorname{dim}_{v}(R)=n$.

(2) $R$ is an integrally closed Mori domain.

(3) $h t_{R}(P)=n$ and $P=\operatorname{rad}_{R}\left(\left(X_{1}, \ldots, X_{m}\right) R\right)$.

(4) $R$ does not satisfy $r$-PIT for $m \leq r \leq n-1$.

(5) $R$ satisfies $r$-PIT for $1 \leq r \leq m-1$.

Proof. We assume that $n<\infty$; the case $n=\infty$ may be treated in a similar manner.

(1) Since $\langle S\rangle=\mathbb{Z}^{n}, \operatorname{dim}(R)=n$ by [8, Theorem 21.4 and Theorem 17.1], while $\operatorname{dim}_{v}(R)=n$ by [2, Theorem 1.17].

(2) Just note that $R=T \cap\left(k+P T_{P}\right)$ (cf. [4, Remark 3.6(c)]).

(3) By $(1), h t_{R}(P) \leq n$. Put $P_{i}=\left(X_{i}, X_{i+1}, \ldots, X_{n}\right) T \cap R$. Since $P=P_{1} \supset P_{2} \supset \cdots \supset P_{n} \supset\{0\}$ is a chain of $n+1$ distinct prime ideals of $R$, we have $h t_{R}(P)=n$. To show that $P=$ $\operatorname{rad}_{R}\left(\left(X_{1}, \ldots, X_{m}\right) R\right)$, it suffices to show that any prime ideal $Q$ of $R$ which contains $X_{1}, \ldots, X_{m}$ also contains $P$. If $f \in P$, then $f^{2} \in\left(X_{1}, \ldots, X_{m}\right) R \subset Q$, and so $f \in Q$. Hence $P=Q$.

(4) This is an immediate consequence of (3). 
(5) Suppose that $1 \leq r \leq m-1$. Let $Q$ be a prime ideal of $R$ minimal over $\left(f_{1}, \ldots, f_{r}\right)$ for some $f_{1}, \ldots, f_{r} \in R$. We show that $h t(Q) \leq r$. If $X_{i} \notin Q$ for some $1 \leq i \leq m$, then $R\left[X_{i}^{-1}\right]=T\left[X_{i}^{-1}\right]$ satisfies GPIT because it is Noetherian, and so $h t(Q)=h t\left(Q\left[X_{i}^{-1}\right]\right) \leq$ $r$. Thus we may assume that $\left(X_{1}, \ldots, X_{m}\right) R \subset Q$. Then, by (3), $P=$ $Q$. Thus $P$ is minimal, as a prime ideal of $T$, over $\left(f_{1}, \ldots, f_{r}\right) T$. Since $h t_{T}(P)=m>r$, this contradicts the fact that $T$ satisfies GPIT.

5. A Krull domain not satisfying GPIT. A celebrated theorem of Mori-Nagata [13, Theorem 33.10(1)] asserts that the integral closure of a Noetherian domain must be a Krull domain. Thus, by Corollary 2.3, each Krull domain arising in this way must satisfy GPIT. As shown in Remark 4.4(b), group algebras lead to other examples of Krull domains satisfying GPIT. Moreover, each Krull domain of dimension $\leq 2$ satisfies (G)PIT. We next present an example of a three-dimensional Krull domain that does not satisfy GPIT. (The history of this example was explained in the introduction.) Finally, as a consequence, we show in Example 5.2 that the class of domains satisfying PIT is not stable under factoring out principal prime ideals.

EXAMPLE 5.1. There exists a quasilocal three-dimensional Krull domain whose maximal ideal is the radical of a 2-generated ideal. Thus, this domain does not satisfy GPIT.

Proof. Following Rees [14], we take $(R, M)$ to be a two-dimensional integrally closed (Noetherian) local domain having a height 1 prime ideal $P$ such that $R / P$ is a DVR and $P$ is not the radical of a principal ideal. (For instance, $R$ can be built from a nonsingular cubic curve over the complex numbers, say $R=\mathbb{C}[x, y, z]_{(x, y, z)}$ where $x^{3}+y^{3}+z^{3}=0$.) As usual let $P^{(n)}=P^{n} R_{P} \cap R$ denote the $n$th symbolic power of $P$. Set

$$
B=R\left[t^{-1}, P t, \ldots, P^{(n)} t^{n}, \ldots\right],
$$

the symbolic Rees ring with respect to $P$. It is known that $B$ is a Krull domain (by adapting a proof of Rees [14, p. 147]: see [6, Lemma 2.1]), non-Noetherian (cf. [6, Theorem 2.2]) and three-dimensional. Observe that $B$ is a $\mathbb{Z}$-graded domain: the homogeneous terms of degree $n$ constitute $P^{(n)} T^{n}$ if $n>0$, and $R t^{n}$ if $n \leq 0$. We shall show that

$$
N=\left(M, t^{-1}, P t, \ldots, P^{(n)} t^{n}, \ldots\right)
$$

is a height 3 (maximal) ideal of $B$ and is the radical of a 2-generated ideal. It will follow that $B_{N}$ has the properties asserted in the statement of Example 5.1. 
Consider the multiplicatively closed set $S=R \backslash P$. Since $R_{P}$ is a DVR, we have $B_{S}=R_{P}\left[t^{-1}, \pi t\right]$, where $\pi \in P \backslash P^{(2)}$. Identify

$$
B_{S}=R_{P}[u, v] /(u v-\pi) .
$$

Evidently, $B_{S}$ is a two-dimensional graded domain with maximal graded ideal $\left(t^{-1}, \pi t\right)$ having height 2 . Hence, $h t_{B}(N)=3$. It remains only to show that $N$ is the radical of a 2-generated ideal.

Next, consider the graded domain $A=B /\left(t^{-1}\right)=R / P \oplus P / P^{(2)} \oplus$ $\cdots \oplus P^{(n)} / P^{(n+1)} \oplus \cdots$. Let $K=R_{P} / P R_{P}$, the quotient field of $R / P$. Since localization commutes with the formation of factor rings, we can identify

$$
A_{S} \cong R_{P}[u, v] /(u, u v-\pi)=R_{P}[u, v] /(u, \pi) \cong K[v],
$$

a polynomial ring in one variable over $K$. Observe that $v$ in $K[v]$ has been identified with the canonical image of $\pi t$ in $A_{S}$. We thus have

$$
(R / P)[v] \subset A \subset K[v]
$$

and $A=\bigoplus F_{n} v^{n}$, where each $F_{n}$ is a fractional ideal of $R / P$. Let $\bar{x}=x+P$ generate the maximal ideal of $R / P$ (where $x \in M)$. We shall show that the maximal graded ideal of $A$, namely $N /\left(t^{-1}\right)$, is the radical in $A$ of $\bar{x} A$. It will follow that $N=\operatorname{rad}_{B}\left(t^{-1}, x\right)$, as desired.

It suffices to show that $F_{n} v^{n} \subset \operatorname{rad}(\bar{x} A)$ for each $n \geq 1$. We claim that there exists $m \geq 1$ such that $\bar{x}^{-1}\left(F_{n} v^{n}\right)^{m} \subset F_{n m} v^{n m}$. If not, then $\left(F_{n}\right)^{m}=F_{n m}$ for each $m \geq 1$. Since $F_{i}=\left(\bar{x}^{-d_{i}}\right)$ for some nondecreasing sequence $\left\{d_{i}\right\}$, a calculation shows that $A=$ $(R / P)\left[\left\{F_{i} v^{i}: 1 \leq i \leq n\right\}\right]$. This contradicts the fact that $A$ is not Noetherian, and thus proves the claim. Then $\left(F_{n} v^{n}\right)^{m} \subset \bar{x} A$, whence $F_{n} v^{n} \subset \operatorname{rad}(\bar{x} A)$. $\S 3$.

The preceding example now leads to an application in the spirit of

ExAmple 5.2. There exists a domain $B$ satisfying PIT and a principal prime ideal $b B$ such that $B / b B$ does not satisfy PIT. It can be arranged that $B$ is a three-dimensional Krull domain.

Proof. Let $B$ be as in Example 5.1 and put $b=t^{-1}$. Then $B / b B=$ $A$ in the above notation. We showed in the proof of Example 5.1 that $\bar{N}=N /\left(t^{-1}\right)=\operatorname{rad}_{A}(\bar{x} A)$; thus, $N$ is minimal over $\bar{x} A$. It remains only to observe that $h t(\bar{N})=2$. 
We have seen in Example 5.1 that not all Krull domains exhibit the Noetherian-like behavior of satisfying GPIT. Another instance of this phenomenon is provided by Fujita's example [7] of a threedimensional noncatenarian UFD. Indeed, it is easy to show that any three-dimensional UFD satisfying GPIT must be catenarian.

Finally, we turn to other Noetherian-like behavior. As in [2], we say that a domain $R$ is a Jaffard domain in case $\operatorname{dim}_{v}(R)=\operatorname{dim}(R)<\infty$. It is an open problem to compute the valuative dimension of an arbitrary Krull domain. With respect to Corollary 4.3 and Example 4.5, it should be noted via [2, Corollary 1.19] that $k[G]$ is a Jaffard domain for each field $k$ and each finite-rank $G$ as in Corollary 4.3. Also, the finite-dimensional Krull domains of Anderson-Mulay [1, (4)] are all Jaffard domains.

In closing, we ask whether the Krull domains that occur in the (generalized) 14th problem of Hilbert must be Jaffard domains. Specifically, let $k$ be a field and let $T$ be either a polynomial ring in $n$ indeterminate over $k$, or (more generally) a normal affine domain over $k$ or a localization of such a domain. Let $F$ be a field between $k$ and the quotient field of $T$, and set $R=T \cap F$. Does it follows that $R$ is a Jaffard domain?

\section{REFERENCES}

[1] D. F. Anderson and S. B. Mulay, Non-catenary factorial domains, Comm. Algebra (to appear).

[2] D. F. Anderson, A. Bouvier, D. E. Dobbs, S. Kabbaj and M. Fontana, On Jaffard domains, Expositiones Math., 6 (1988), 145-175.

[3] D. F. Anderson and D. E. Dobbs, Pairs of rings with the same prime ideals, II, Canad. J. Math., (to appear).

[4] V. Barucci, D. F. Anderson and D. E. Dobbs, Coherent Mori domains and the principal ideal theorem, Comm. Algebra, 15 (1987), 1119-1156.

[5] E. D. Davis, Overrings of commutative rings. III: Normal pairs, Trans. Amer. Math. Soc., 182 (1973), 175-185.

[6] P. Eakin and W. Heinzer, Non-finiteness in finite dimensional Krull domains, J. Algebra, 14 (1970), 333-340.

[7] K. Fujita, Three-dimensional unique factorization domain which is not catenary, J. Algebra, 49 (1977), 411-414.

[8] R. Gilmer, Commutative semigroup rings, in Chicago Lectures in Mathematics, Univ. of Chicago Press, Chicago and London, 1984.

[9] _ A two-dimensional non-Noetherian factorial ring, Proc. Amer. Math. Soc., 44 (1974), 25-30.

[10] R. W. Gilmer and W. J. Heinzer, Intersections of quotient rings of an integral domain, J. Math. Kyoto Univ., 7 (1967), 133-150.

[11] A. Grothendieck, Eléments de Géométrie Algébrique, Chapitre IV (Première Partie), Publ. Inst. Hautes Etudes Sci., no. 20 (1964). 
[12] I. Kaplansky, Commutative Rings, revised edition, Univ. of Chicago Press, Chicago, 1974.

[13] M. Nagata, Local Rings, Interscience, New York, 1962.

[14] D. Rees, On a problem of Zariski, Illinois J. Math., 2 (1958), 145-149.

Received November 30, 1988 and in revised form April 24, 1989. The first author was supported in part by a National Security Agency Grant. The second author was supported in part by a University of Tennessee Faculty Research Grant. The fourth author was supported in part by NSF Grant DMS-8521767 and the University of Tennessee Science Alliance.

UNIVERSITY OF TENNESSEE

KNOXVILLE, TN 37996-1300

UNIVERSITY OF TENNESSEE

KNOXVILLE, TN 37996-1300

UNIVERSITY OF KENTUCKY

LEXINGTON, KY 40506

AND

Purdue University

West LAFAyETTE, IN 47907 



\section{PACIFIC JOURNAL OF MATHEMATICS EDITORS}

\author{
V. S. VARADARAJAN \\ (Managing Editor) \\ University of California \\ Los Angeles, CA 90024-1555-05 \\ Herbert Clemens \\ University of Utah \\ Salt Lake City, UT 84112 \\ Thomas EnRIght \\ University of California, San Diego \\ La Jolla, CA 92093
}

R. FINN

Stanford University

Stanford, CA 94305

HeRmanN FlaschKa

University of Arizona

Tucson, AZ 85721

VAughan F. R. Jones

University of California

Berkeley, CA 94720

Steven Kerckhoff

Stanford University

Stanford, CA 94305

\section{C. Moore}

University of California

Berkeley, CA 94720

Martin ScharlemanN

University of California

Santa Barbara, CA 93106

Harold Stark

University of California, San Diego

La Jolla, CA 92093

\section{ASSOCIATE EDITORS}
R. ARENS
E. F. BECKENBACH
B. H. NEUMANN
F. WoLF
K. YoshidA (1906-1982)
(1904-1989)

\section{SUPPORTING INSTITUTIONS}

UNIVERSITY OF ARIZONA
UNIVERSITY OF BRITISH COLUMBIA
CALIFORNIA INSTITUTE OF TECHNOLOGY
UNIVERSITY OF CALIFORNIA
MONTANA STATE UNIVERSITY
UNIVERSITY OF NEVADA, RENO
NEW MEXICO STATE UNIVERSITY
OREGON STATE UNIVERSITY

\author{
UNIVERSITY OF OREGON \\ UNIVERSITY OF SOUTHERN CALIFORNIA \\ STANFORD UNIVERSITY \\ UNIVERSITY OF HAWAII \\ UNIVERSITY OF TOKYO \\ UNIVERSITY OF UTAH \\ WASHINGTON STATE UNIVERSITY \\ UNIVERSITY OF WASHINGTON
}

The Supporting Institutions listed above contribute to the cost of publication of this Journal, but they are not owners or publishers and have no responsibility for its content or policies.

Mathematical papers intended for publication in the Pacific Journal of Mathematics should be in typed form or offset-reproduced (not dittoed), double spaced with large margins. Please do not use built up fractions in the text of the manuscript. However, you may use them in the displayed equations. Underline Greek letters in red, German in green, and script in blue. The first paragraph must be capable of being used separately as a synopsis of the entire paper. In particular it should contain no bibliographic references. Please propose a heading for the odd numbered pages of less than 35 characters. Manuscripts, in triplicate, may be sent to any one of the editors. Please classify according to the 1980 Mathematics Subject Classification (1985 Revision) scheme which can be found in the December index volumes of Mathematical Reviews. Supply name and address of author to whom proofs should be sent. All other communications should be addressed to the managing editor, or Elaine Barth, University of California, Los Angeles, California 90024-1555-05.

There are page-charges associated with articles appearing in the Pacific Journal of Mathematics. These charges are expected to be paid by the author's University, Government Agency or Company. If the author or authors do not have access to such Institutional support these charges are waived. Single authors will receive 50 free reprints; joint authors will receive a total of 100 free reprints. Additional copies may be obtained at cost in multiples of 50 .

The Pacific Journal of Mathematics (ISSN 0030-8730) is published monthly. Regular subscription rate: $\$ 190.00$ a year (12 issues). Special rate: $\$ 95.00$ a year to individual members of supporting institutions.

Subscriptions, orders for numbers issued in the last three calendar years, and changes of address should be sent to Pacific Journal of Mathematics, P.O. Box 969, Carmel Valley, CA 93924, U.S.A. Old back numbers obtainable from Kraus Periodicals Co., Route 100, Millwood, NY 10546.

The Pacific Journal of Mathematics at P.O. Box 969, Carmel Valley, CA 93924 (ISSN 0030-8730) is published monthly. Second-class postage paid at Carmel Valley, California 93924, and additional mailing offices. Postmaster: send address changes to Pacific Journal of Mathematics, P.O. Box 969, Carmel Valley, CA 93924.

PUBLISHED BY PACIFIC JOURNAL OF MATHEMATICS, A NON-PROFIT CORPORATION

Copyright (C) 1990 by Pacific Journal of Mathematics 


\section{Pacific Journal of Mathematics}

\section{Vol. 146, No. 2 December, 1990}

David Fenimore Anderson, David Earl Dobbs, Paul M. Eakin, Jr. and

William James Heinzer, On the generalized principal ideal theorem and Krull domains .................................... 201

Richard C. Bradley, On $\rho$-mixing except on small sets $\ldots \ldots \ldots \ldots \ldots 217$

David H. Collingwood and Brad Shelton, A duality theorem for extensions

of induced highest weight modules $\ldots \ldots \ldots \ldots \ldots \ldots \ldots \ldots \ldots \ldots 27$

Gerd H. Fricke and John Albert Fridy, Sequence transformations that guarantee a given rate of convergence $\ldots . \ldots \ldots \ldots \ldots \ldots \ldots . \ldots . \ldots . \ldots 239$

Michael Justin Heumos and Stephen James Rallis, Symplectic-Whittaker

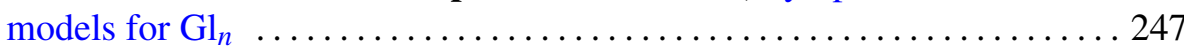

Severino Toscano do Rego Melo, A comparison algebra on a cylinder with

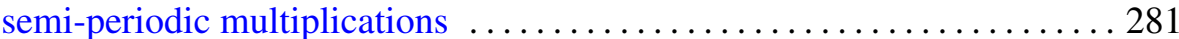

Daniel Pecker, On the elimination of algebraic inequalities ........... 305

Geoffrey Lynn Price, The $C^{*}$-algebras generated by pairs of semigroups of isometries satisfying certain commutation relations $\ldots \ldots \ldots \ldots \ldots \ldots 315$

John Kurt Sauter, Jr., Isomorphisms among monodromy groups and

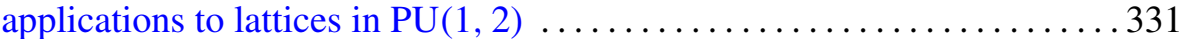

Shoji Yokura, A formula for Segre classes of singular projective varieties . . 385 